\title{
Brachytherapy Doses and Late Rectal and Bladder Toxicities for Cervical Cancer Patients: A Case Study of a Radiotherapy Centre in Zambia
}

\author{
Ernest Chanda1*, Professor Godfrey Azangwe ${ }^{2}$, Osward Bwanga ${ }^{3}$ \\ ${ }^{1}$ Cancer Diseases Hospital, Radiotherapy Department, Zambia \\ ${ }^{2}$ National University of Science and Technology, Zimbabwe \\ 3 Midland Regional Hospital at Tullamore, Radiology Department, Ireland
}

\section{${ }^{*}$ Corresponding Author \\ Ernest Chanda}

\section{Article History}

Received: 12.07.2019

Accepted: 22.07.2019

Published: 30.07 .2019

\begin{abstract}
The purpose of this study was to analyse the International Commission of Radiation Unit (ICRU) point doses delivered to the urinary bladder and rectum with ultrasound based intracavitary brachytherapy and to evaluate late toxicity relative to the equivalent total doses delivered in 2 gray per fraction of equivalent dose (EQD2) for bladder and rectum using point based methods of reporting. This study was conducted using a quantitative retrospective research design which looked at three hundred and thirty three women who received external beam radiation therapy (EBRT) + brachytherapy (BT) between 2009 to 2013. The patients were aged 25 years and above the median age was 48 . The median follow-up was 24 months and $58 \%$ of patients received 50 Gray in 25 fractions with 24 Gray in 3 fractions of BT and $42 \%$ received 46 Gray in 23 fractions to the pelvis with 26 Gray in 4 fractions of BT. BT was delivered with ring and tandem applicators. The results showed no correlation between late toxicity for rectum and total EQD2 to ICRU rectal points $r_{s}=0.1$. For bladder the null hypothesis that there was no association between EQD2 and late toxicity grade for bladder was rejected and the alternative hypothesis that there was association between EDQ2 and late toxicity grade for bladder accepted, $r_{s}=0.013$. The mean rectal and urinary bladder doses at ICRU points were $59.8 \%$ (4.5 Gy) and 62\% (4.6 Gy) which was below the ICRU 38 recommended dose of lower than $80 \%$ of maximum dose to the rectum and bladder of the prescribed dose to point A.
\end{abstract}

Keywords: Toxicity, Intracavitary brachytherapy, External beam radiotherapy, International commission for radiation units 38 , Point doses, cervical cancer, EQD2- Equivalent total doses delivered in 2 Gray per fraction

\section{INTRODUCTION}

High dose rate (HDR) brachytherapy is an essential component for treatment of locally advanced cervical cancer. It increases the initial doses from external beam radiation therapy (EBRT) up to 80-90 Gray without causing undue side effects to the patients. It is recommended that to achieve local control of the disease brachytherapy should be part of the treatment [1]. However, there are critical organs that are found closer to the cervix and these can easily be affected by these doses and can result in toxicities [2]. Toxicities result in poor quality of life after EBRT and HDR brachytherapy some of these organs that are highly susceptible are urinary bladder and rectum. Brachytherapy is usually recommended after EBRT mostly for all cases of locally advanced cervical cancer ranging from stages of $1 \mathrm{~B} 2$ to IVA [3].

To reduce toxicities after treatment of cervical cancer with EBRT plus HDR, it is of paramount importance that the doses to critical organs, such as the urinary bladder and rectum are monitored during treatment. This measure will allow the treatment team to know if these organs are receiving doses that are exceeding the tolerance doses. Exceeding tolerance doses to the critical organs will result in the likelihood of manifestation of unacceptable side effects, such as dysuria, incontinence,cystitis, fistulas hematuria etc.

To determine the doses to the rectum and urinary bladder, ICRU has recommended ways of measuring these doses [4]. In order to standardize and allow for information exchange of clinical outcomes amongst the cancer institutions some methods of dose reporting have been divised, to specify doses and the volumes to which these doses have been distributed. In recognising the importance of uniformity in reporting the ICRU has come up with concepts and definitions of terms in radiation therapy [4].The bladder

Copyright @ 2019: This is an open-access article distributed under the terms of the Creative Commons Attribution license which permits unrestricted use, distribution, and reproduction in any medium for non commercial use (NonCommercial, or CC-BY-NC) provided the original author and source are credited. 
point is defined as the surface of the foley catheter balloon (filled with $7 \mathrm{cc}$ contrast medium) it's a representation of the maximum dose to the bladder. Rectal point specification is at $0.5 \mathrm{~cm}$ posterior to the vaginal wall [4]. Point A refers to the dose specification in BT using ICRU 38 classification for point based dosimetry owing to the scarcity of 3D imaging global wise, it involves the use of plain radiographs. In this case instead of prescribing the dose to the tumour, dose is prescribed to a point which relates to the locality of the sources and not to any specified anatomical structure [5]. Kim et al. [6] found that there is a direct relationship between the dose delivered to the critical organs in pursuit of treating a uterine cervix lesion and late toxicities that are likely to occur. It is therefore critical that a correlation of the doses to late toxicities in the management of these patients is undertaken.

This study summarises the report on clinical outcomes of late toxicities for rectum and bladder after definitive radiotherapy (radiotherapy given with a chance to cure) in uterine cervix cancer using ICRU reference points as dose predictors as well as the documentation of the pattern of these toxicities at Cancer Diseases Hospital (CDH). Cancer Diseases Hospital is the only hospital providing radiotherapy services in Zambia. In addition, it also provides chemotherapy, diagnostic imaging, laboratory and in-patient care for oncology cases and it is located in the capital city of Lusaka. The radiotherapy department has different machines which includes one linear accelerator, two Cobalt-60 Teletherapy machines, two remote after-loading HDR brachytherapy units, one conventional simulator, a computed tomography (CT), simulator and three (3D) dimension treatment planning system. Cervical cancer patients requiring radical radiotherapy receive EBRT using a Linear accelerator (6 or 10 megavoltage photons) or Cobalt 60 Teletherapy machine. Brachytherapy is administered in order to boost the dose to the cervix and parametria.

In evaluating the success of treatment two important factors must be considered these being, tumour control and acceptable toxicities [7]. These factors are directly dependent on the dose administered. Therefore, any treatment protocol must have empirical evidence that the treatment outcome in terms of disease control and toxicity will be within the accepted standard. Therefore, it's important to correlate dose to the clinical outcomes or late toxicity in particular, doing so will enable the radiation oncologist to analyse the impact of various clinical factors on late rectal and bladder toxicity [1]. CDH uses the ICRU 38 reference points for dose and volume specification for reporting intracavitary therapy in gynecology. ICRU provides guidance on dose reporting in two dimension (2D) brachytherapy. Khan and Gibbons [8] point out that there's a possibility that point A can be in or outside the cervix due to the size of the cervix. As a result dose prescription to point A could lead to the under dosage of large cancers and overdosing the smaller ones as [9].

In brachytherapy it is important to understand the anatomy of the female pelvis, so that the treatment can be planned in such a way that adjacent organs at risk receive minimal radiation. This will help in minimising toxicities in order to maintain a better quality of life after treatment [10]. The organs at risk in the female pelvis are the bowels, anus, vaginal, ovaries, skin inclusive of rectum and urinary bladder which were considered in the study. The aim of the study, therefore, was to investigate brachytherapy doses and late rectal and bladder toxicities for uterine cervical cancer patients treated at CDH of Zambia.

\section{METHODS}

This study was conducted following the application and approval of the research by CDH, and ERES (Ethics Research) converge review board of Zambia. A quantitative research design using a retrospective cross sectional approach was utilised in conducting this study. The quantative research design was chosen because there was a need to show the relationship between the brachytherapy doses and late rectal and urinary bladder toxicities as per objective of this research [11]. A cross sectional study was also used aimed at establishment of the prevalence of these late toxicities as well as establish both cause and effect at the same point. Retrospective approach was chosen in the sense that the issue under study involved gathering data from the use of previous documented data [11].

\section{Population and Sampling}

The target population for this study was all the cervical cancer patients treated with radical intent from 2009-2013. The population was chosen because it had the potential to provide the information for the study. Patients who were treated from this period had enough clinical follow up visits that generated the required information. The number of uterine cervical cancer patients treated in this period was 2507 . The files that were used in the sample size $(\mathrm{N}=333)$ were identified using systematic sampling. Mann [11] defines a systematic sampling as a type of probability sampling method in which sample members from a large population are selected according to a random starting point but with a fixed, periodic interval. This type of sampling was used inoder to provide adequate covering of the population.

The following were inclusion and exclusion criteria for the study.

\section{Inclusion Criteria}

All cervical cancer patients that were treated with radical intent from 2009-2013 at CDH with EBRT and Brachytherapy.

\section{Exclusion Criteria}

All cervical cancer patients who received palliative treatment. $\mathrm{CDH}$ protocol only gives radical treatment to locally advanced disease which includes brachytherapy. For advanced stages patients receive a single dose or $5 / 10$ fractions of EBRT without 
brachytherapy for palliative purposes. Patients also with prior rectal and bladder conditions may have severe effects. These were the reasons for excluding palliative cases from the study.

\section{Data Collection}

The data collected in this study was predominantly quantitative to meet the objective of describing the prevalence and relationship of brachytherapy doses and late rectal and urinary bladder toxicities. A data collection form was designed using the Radiation oncology group (RTOG) toxicity criteria to score the toxicities. The extration form contained age, stage, chemotherapy, haemoglobin, duration, EBRT, biological effective dose, brachytherapy sessions, total dose EBRT, fractionation, type of treatment field AP/PA or Box, type of energy used 6/10MV or Cobalt 60, existing cormobidities (HIV status), ICRU 38 reported rectal doses,cICRU 38 reported bladder doses, total EBRT + BT, Doserate, boost, grade for rectal toxicity and grade for bladder toxicity. Data of 333 patients on Rectal and urinary bladder toxicities who underwent definitive radiotherapy with chemotherapy of uterine cervical cancer was extracted from their files from the period 2009 to 2013 and records of clinical outcomes 6 to 2 years post chemoradiotherapy was recorded.

\section{Data Management and Analysis}

Microsoft excel was used to enter data. Statistical software package namely the Statistical packages for the social sciences (SPSS) version 22 was used. The analysis used descriptive figures, such as percentages and ratios presented in form of graphs, tables and charts. Furthermore hypothesis testing was done using the Spearmans correlation test to ascertain the the relationship between the brachytherapy doses and late rectal and urinary bladder toxicities.

\section{RESULTS}

Three hundred and thirty three women received EBRT + BT and the patients were aged 25 years and above, $63 \%$ were aged $35-54$, followed by $19 \%$ (55-64), 65 years and above $11 \%$ and $7 \%$ represented those from ages $25-34$ median age was 48 (Table 1).

Table-1: Showing the age distribution from the patient files

\begin{tabular}{|c|c|c|}
\hline Age group (Years) & Frequency & Percentage \\
\hline 25 to 34 & 22 & 6.6 \\
\hline 35 to 44 & 106 & 31.8 \\
\hline 45 to 54 & 105 & 31.5 \\
\hline 55 to 64 & 63 & 18.9 \\
\hline 65 to 74 & 29 & 8.7 \\
\hline 75 and above & 8 & 2.4 \\
\hline Total & 333 & 100.0 \\
\hline
\end{tabular}

The study results of the 333 patient records reviewed, the women were all aged 25 and above. In terms of the distribution by age group $31.8 \%$ (106) were aged $35-44,31.5 \%$ (105) aged 45-54, 18.9\% (63) were aged 55-64, 8.7\% (29) were aged 65-74, 6.6\% (22) aged $25-34$, and $2.4 \%$ (8) were aged 75 and above. The mean age was 49.14 years while the median age was 48 years. The mean hemoglobin level of the from the patient files reviewed was $11.1 \mathrm{hb} / \mathrm{gdl}$.

\section{Constant Values Used during the Research}

The constant variables used for calculating EQD2 in this study were EQD2EBRT, BEDBRT, alpha-beta ratio and fractionation. The respective values of these were 2.00, 3.30, 3.00 and 2.00 gy.

\section{Reported Mean BT Doses for Rectal and Bladder at ICRU Reference Points}

The calculated mean brachytherapy rectal and bladder doses at ICRU - B and ICRU - R point for CDH from 2009-2013 was $59.8 \%$ representing $(4.5 \mathrm{~Gy}$ ) and $61.9 \%$ (4.6 Gy) respectively. 


\section{Tumour stage}

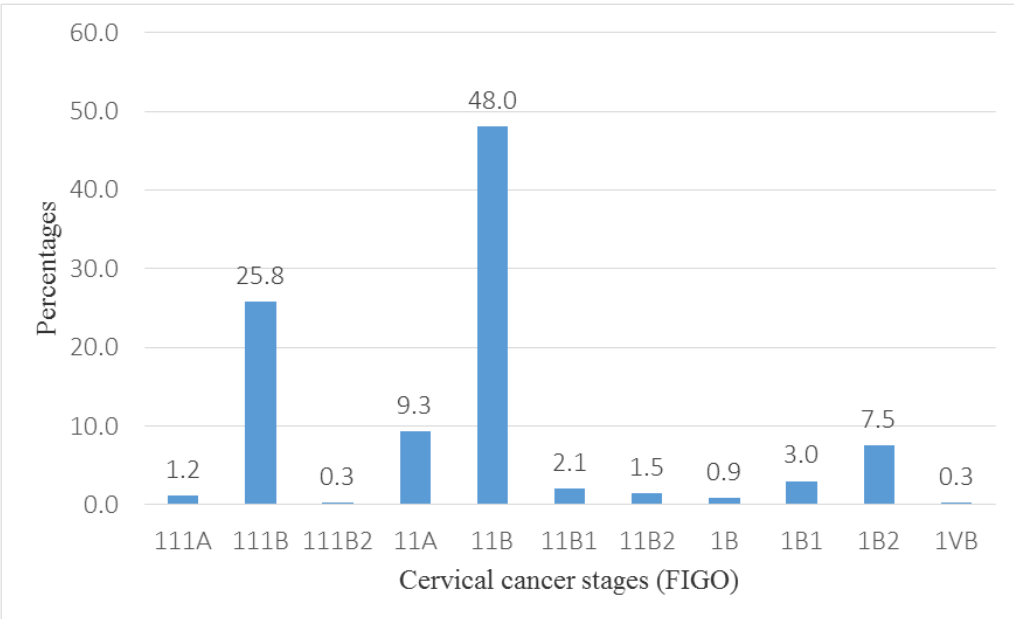

Fig-1: Distribution of patients with different stages of the disease

Figure 1 shows that the stage of the tumours have a significant number $(48 \%)$ of patients were in stage $11 \mathrm{~B}$, followed by those in $111 \mathrm{~B}$ at $25.8 \%$, then stage $11 \mathrm{~A}$ at $9.3 \%, 1 \mathrm{~B} 2$ at $7.5 \% 1 \mathrm{~B} 1$ at $3 \%, 11 \mathrm{~B} 1$ at $2.1 \%, 11 \mathrm{~B} 2$ at $1.5 \%, 111 \mathrm{~A}$ at $1.2 \%, 1 \mathrm{~B}$ at $0.9 \%$ then 111B2 and $1 \mathrm{VB}$ at 0.3 each.

\section{Chemotherapy Versus no Chemotherapy}

The majority (78\%) of the patients received chemotherapy, whereas the other $22 \%$ did not receive.

\section{Duration of EBRT} 23 fractions.

The findings showed that slightly above half $(57.7 \%)$ of the patients received 25 fractions of EBRT, whereas $42.3 \%$ received

\section{Dose per fraction BT} BT.

The study showed that $57.7 \%$ of the patients received 8 gy per fraction of bt, whereas $42.3 \%$ received 6.5 gy per fraction of

\section{BT sessions}

The research results showed that $57.1 \%$ of the patients received 3 sessions BT, wVhereas $42.9 \%$ received 4 BT sessions.

\section{AP/PA or box fields techniques}

The research findings showed that most (95\%) of the patients were treated using the ap/pa, whereas only $5 \%$ were treated using the box field technique.

\section{Existing co-morbidities}

The study results showed $61 \%$ of the patients were hiv non-reactive, whereas $39 \%$ were hiv-reactive.

\section{Energies used} $10 \mathrm{MV}$

The energies used, $55 \%$ of the patients were treated on Cobalt- 60 , followed by $30 \%$ who were treated on $6 \mathrm{MV}$, and $15 \%$ on 


\section{EDQ2 total EBRT plus BT}

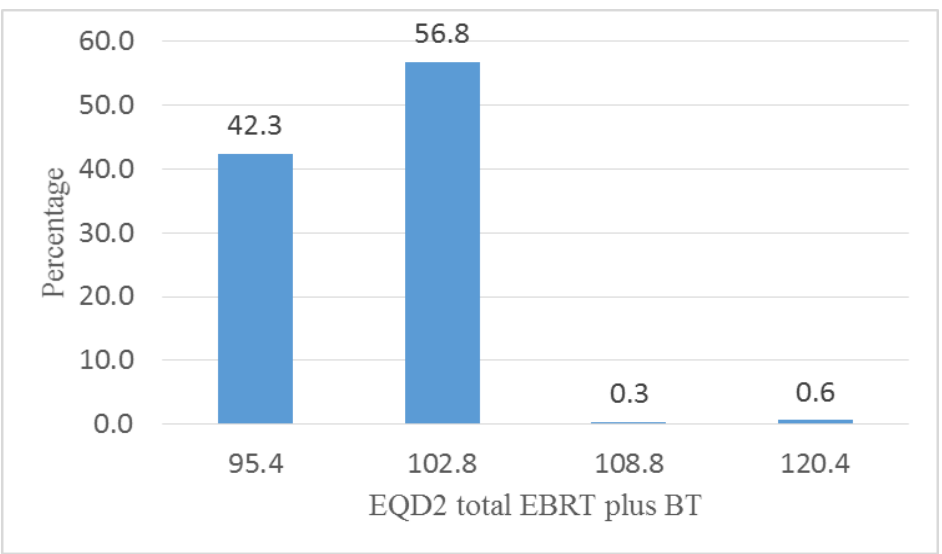

Fig-2: Showing EDQ2 Total EBRT plus BT

Figure 2 shows number of patients who developed late toxicities at each level of EQD2 plus EBRT + BT within six months to two years post BT+EBRT from 2009-2013.

\section{Late toxicity for rectum}

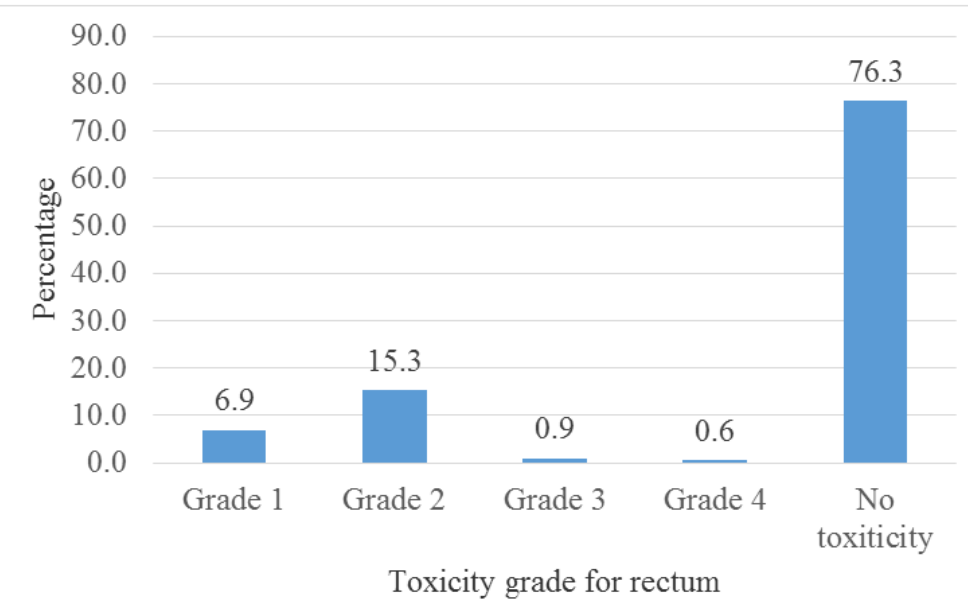

Fig-3: Showing Patients who presented with late toxicity for rectum

According to the study findings illustrated in figure 3, the majority of the patients $(76.3 \%)$ of the patients did not present with any late rectal toxicity within six month to two years post BT+EBRT from 2009-2013, however, $15.3 \%$ presented with Grade 2 late toxicity, $6.9 \%$ presented with Grade 1 late toxicity, $0.9 \%$ with Grade 3 late toxicity, and $0.6 \%$ with Grade 4 late toxicity.

\section{Late toxicity for urinary bladder}

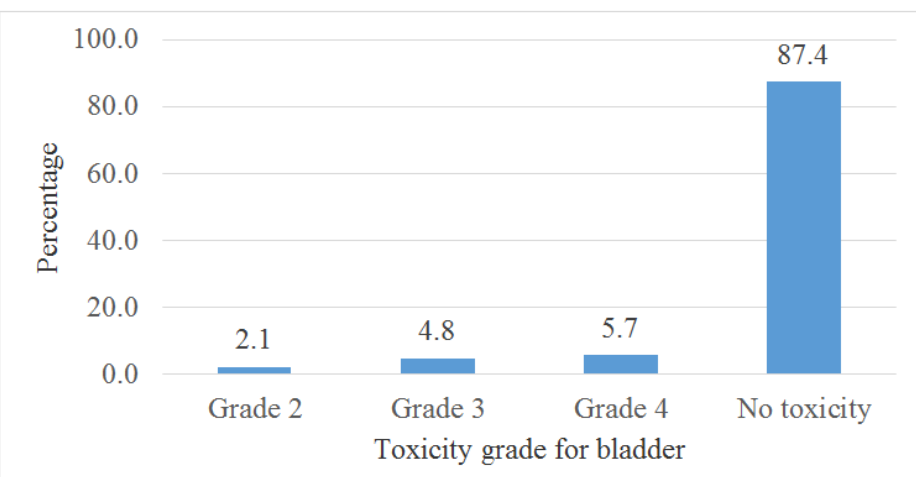

Fig-4: Showing Patients who presented with late toxicity for bladder 
Study findings illustrated in figure 4 , shows that the majority of the patients $(87.4 \%)$ of the patients did not present with any late bladder toxicity within six months to two years post BT+ EBRT from 2009-2013, on the other hand, $5.7 \%$ presented with grade 4 late toxicity $4.8 \%$ presented with grade 3 late toxicity, $2.1 \%$ with grade 2 late toxicity, and none presented with grade 1 late toxicity.

\section{Late toxicity post bt+ebrt for rectum}

Table-2: Showing the relationship of late toxicity and EQD2 post BT+EBRT for rectum

\begin{tabular}{|l|l|l|l|l|l|}
\hline \multirow{2}{*}{ Toxicity Grade for rectum } & \multicolumn{5}{|l|}{ EDQ2TotaIEBRTplusBT } \\
\cline { 2 - 6 } & 95.40 & 102.80 & 108.80 & 120.40 & Total \\
\hline Grade 1 & 8 & 15 & 0 & 0 & 23 \\
\hline Grade 2 & 16 & 34 & 0 & 1 & 51 \\
\hline Grade 3 & 2 & 1 & 0 & 0 & 3 \\
\hline Grade 4 & 1 & 1 & 0 & 0 & 2 \\
\hline No toxicity & 114 & 138 & 1 & 1 & 254 \\
\hline Total & 141 & 189 & 1 & 2 & 333 \\
\hline
\end{tabular}

Table 2 depicts the relationship between late toxicity for rectum and EDQ2 total EBRT+BT.

According to the results, in table only 79 patients out of the 333 patients presented with late toxicities from six months to two years. At EDQ2 95.4 gray; there were 16 patients with late toxicity grade 2, 8 patients with late toxicity grade 1, 2 patients with late toxicity grade 3 and 1 patient with late toxicity grade 4. at EDQ2 102.8 gray; there were 34 patients with late toxicity grade 2, 15 patients with late toxicity grade 1 , and 1 each for late toxicity grade 3 and grade 4 . on the other hand there was no late toxicity recorded at EDQ2 108.8 gray. However, there was 1 grade 2 late toxicity at EDQ2 120.4 gray.

\section{Late toxicity post BT+EBRT for bladder}

Table-3: Showing the relationship of late toxicity and EQD2 post BT+EBRT for bladder

\begin{tabular}{|l|l|l|l|l|l|}
\hline \multirow{2}{*}{ Toxicity Grade for bladder } & \multicolumn{5}{|l|}{ EDQ2TotalEBRTplusBT } \\
\cline { 2 - 6 } & 95.40 & 102.80 & 108.80 & 120.40 & Total \\
\hline Grade 2 & 2 & 5 & 0 & 0 & 7 \\
\hline Grade 3 & 3 & 13 & 0 & 0 & 16 \\
\hline Grade 4 & 5 & 14 & 0 & 0 & 19 \\
\hline No toxicity & 131 & 157 & 1 & 2 & 291 \\
\hline Total & 141 & 189 & 1 & 2 & 333 \\
\hline
\end{tabular}

Table 3 above depicts the relationship between late toxicity for bladder and EDQ2 total EBRT+BT.

According to the results, only $42(12.6 \%)$ patients out of the 333 patients presented with late toxicities from six (6) months to two (2) years. At EDQ2 95.40 gray; there were 5 patients with late toxicity grade 4, 3 patients with late toxicity grade 3, 2 patients with late toxicity grade 4. At EDQ2 102.80 gray; there were 14 patients with late toxicity grade 4, 13 patients with late toxicity grade 3, and 5 with late toxicity grade 2 . On the other had 291 patients did not record any late toxicities.

\section{Late toxicity post BT+EBRT for rectum}

Table-4: Showing the relationship of late toxicity and EQD2 post BT+EBRT for rectum

\begin{tabular}{|l|c|c|c|c|c|}
\hline \multirow{2}{*}{ Toxicity Grade for rectum } & \multicolumn{5}{|c|}{ EDQ2TotalEBRTplusBT } \\
\cline { 2 - 6 } & 95.40 & 102.80 & 108.80 & 120.40 & Total \\
\hline Grade 1 & 8 & 15 & 0 & 0 & 23 \\
\hline Grade 2 & 16 & 34 & 0 & 1 & 51 \\
\hline Grade 3 & 2 & 1 & 0 & 0 & 3 \\
\hline Grade 4 & 1 & 1 & 0 & 0 & 2 \\
\hline No toxicity & 114 & 138 & 1 & 1 & 254 \\
\hline Total & 141 & 189 & 1 & 2 & 333 \\
\hline
\end{tabular}

Table 4 depicts the relationship between late toxicity for rectum and EDQ2 total EBRT+BT. According to the results, in figure 23 only 79 patients out of the 333 patients presented with late toxicities from six months to two years. At EDQ2 95.4 gray; there were 16 patients with late toxicity grade 2, 8 patients with late toxicity grade 1,2 patients with late toxicity grade 3 and 1 patient with late toxicity grade 4. At EDQ2 102.8 gray; there were 34 patients with late toxicity grade 2, 15 patients with late toxicity grade 1, and 1 each for late toxicity grade 3 and grade 4 . On the other hand there was no late toxicity recorded at EDQ2 108.8 gray. However, there was 1 grade 2 late toxicity at EDQ2 120.4 gray. 
Table-5: Table showing correlation of the EDQ2 for EBRT plus BT and late toxicity grade for rectum and bladder

\begin{tabular}{|l|c|c|}
\hline & Rectum & Bladder \\
\hline Spearman's rho EDQ2TotalEBRTplusBT Correlation Coefficient Sig. (2-tailed) & 0.100 & 0.013 \\
\hline $\mathrm{N}$ & 333 & 333 \\
\hline
\end{tabular}

Table 5 shows no correlation between EQD2 (EBRT + BT) and late rectal toxicities, and the study also failed to reject the alternative hypothesis that there was association between EDQ2 and late toxicity grade for bladder.

\section{DISCUSSION}

The discussion is done in relation to the findings of this study and existing literature.

\section{Age Profile}

There were more patients in the age group (35-54 years) representing $63 \%$ who were treated for uterine cervical cancer and the median age was 48 years. This number resonates with what is in literature that cervical cancer is more prevalent in this ages. In an article published by Gattoc et al. [13] indicated that the ultimate age of developing cervical cancer was at 47 years. The American cervical cancer facts and statistics reported that the median age of cervical cancer diagnosis from 2006-2012 was 49 years which was within the study findings which had the median age of 48 years [24].

\section{Stage Distribution}

In the study the distribution of tumour stage was that a significant number of patients were in stage $11 \mathrm{~B}(48 \%)$, followed by those in $111 \mathrm{~B}$ at $25.8 \%$, then stage $11 \mathrm{~A}$ at $9.3 \%$, and $1 \mathrm{~B} 2$ at $7.5 \%$ the rest of the stages accounted for $9 \%$. This is in agreement with the study done by Logar $\mathrm{HB}$ et al. [14] which also had a significant numbers of age distribution in stage IIB and IIIB at $37 \%$ and $42 \%$ respectively then followed by $10 \% \mathrm{IB}, 7 \% \mathrm{IIA}$ and $4 \%$ IIIA. This analysis was fundamental in order to determine the magnitude of late presentation for radiotherapy by cervical cancer patients at $\mathrm{CDH}$, Zambia. These results meant that most of the cervical cancer patients had locally advanced disease.

\section{Field Orientation (AP/PA OR BOX)}

Field orientation determines beam weighting the field with more beams is likely to have less toxicities than the field with fewer beam arrangements [15]. The research finding showed that (95\%) of the patients were treated using the AP/PA, whereas only $5 \%$ were treated using the box field technique.

\section{Cormobidity ( HIVstatus)}

The study showed that the incidence of late toxicity in both bladder and rectum had no relation with the HIV status of the patient, for instance the rectum had $16.3 \%$ reactive patients who had developed grade 2 toxicity and $13.8 \%$ non reactive patients with grade 2 toxicity as well. For bladder $5.4 \%$ non reactive patients had grade 3 while $3.8 \%$ with same grade were non reactive.

\section{Energies Used}

Photons in the ranges of $10 \mathrm{MV}$ and higher are capable of treating deep seated tumours owing to their high penetrating ability in the process sparing the normal tissue. However in clinical practise the exit dose is substantially high posing a risk of overdosing the normal tissue, as well as the ability of high energies of photon to produce secondary electron resulting in penumbra that results in overdosing of normal critical structures. The reduced penumbra is capable to deliver close-fitting dose distribution closer to the target. In this lower energies there is also low exit dose which is beneficial to the critical organs closer to the target [16]. More patients in this study were treated with cobalt $60(55 \%)$ as compared to $6 \mathrm{MV}(30 \%)$ and $10 \mathrm{MV}(15 \%)$ reason being that when $\mathrm{CDH}$ commenced treatment the cobalt unit was the only unit that was functional but later the linear acceralator which had 6 MV and 10 MV was introduced.

\section{Chemotherapy}

Chemotherapy as an influence on acute toxicity and not on late toxicity reason being that late toxicity is dose dependant [17].There were $(78 \%)$ of the patients who received chemotherapy, whereas the other $22 \%$ did not receive.

\section{Age with Late Toxicities}

Age and late toxicities revealed that age was not a factor in the development of late toxicities as there was random distribution of the toxicities regardless of the age from 25 to 75 years and above. The trend was that toxicities were not varying with age. For instance grade 2 bladder toxicity had $2.9 \%$ patients in the age group of $45-54$ and $3.2 \%$ patients in the age group of $55-64$ and for grade 2 rectal toxicity $13.6 \%$ in age group $25-34$ and $10.3 \%$ in age group 65-74. Bladder grade 3 also for ages $25-34$ it was $4.5 \%$ and $3.2 \%$ for ages 55 to 64 years.

\section{Stage with Late Toxicities}

For stage and late toxicities only grade 4 bladder toxicity showed that they was an increase in toxicities with an increase in 
stage. For grade 4 : there were $(10.5 \%)$ patients with stage $111 \mathrm{~B},(5 \%)$ with $11 \mathrm{~B}$, and $(4 \%)$ for stages $1 \mathrm{~B} 2$ and $(3.2 \%)$ for $11 \mathrm{~A}$. In the rest of the grades for bladder, there was random distribution of toxicities with stage.The relationship between late toxicity grade for rectum and the stage completely showed no relationship as they was random distribution of toxicities regardless the stage of the tumour for grade 1 were $(10.5 \%)$ patients with stage $111 \mathrm{~B},(5 \%)$ patients with stage $11 \mathrm{~B},(6.5 \%)$ patients at stage $11 \mathrm{~A}$ and $(8 \%)$ at $1 \mathrm{~B} 2$ and $(10 \%)$ at $1 \mathrm{~B} 1$. This findings are not in line with the study done by [6] which showed that late toxicities were more in the patients with stage $11 \mathrm{~B}$ and 111 compared to those in early stages.

\section{Field Orientation and Late Toxicity}

The field orientation and late toxicity grade for rectum and bladder had a fairly distribution of toxicity grades for both AP/PA and Box field for instance all the $0.6 \%$ patients who had rectal grade 4 were treated using AP/PA. For bladder grade 4 AP/PA had almost the same range of toxicity at $(5.7 \%)$ with box technique which had $(6.3 \%)$. The trend of not having bias to one type of orientation to produce more toxicities was evident in all the other grades.

Though in literature AP/PA fields have the pontential to induce bowel toxicity for instance in patients with history of hysterectomy, the small bowel tends to move in the pelvic region [18]. The use of box field is an alternative to AP/PA to help in reducing toxicities to the critical organs during pelvic irradiation [15]. As a result more toxicities were expected in AP/PA fields than in box fields. The use of multiple photon beam radiation fields helps to achieve a uniform dose distribution in the target volume and minimise the dose as low as possible in organs at risk [19].

\section{HIV Status and Late Toxicity}

There are scarce studies that have looked at late toxicities in HIV reactive and non reactive patients after definitive radiotherapy of cervical cancer. The study done by [20] on acute toxicity showed that they was no increase in toxicity in HIV reactive patients more than they are in non reactive HIV patients. As long the patient was on HAART they was no difference interms of acute toxicity. However in this study on crosstabulation there were more HIV negative patients who developed late toxicities than the HIV reactive ones. Except for grade 1 rectal toxicity were the reactive ones were at $9.2 \%$ compared to the $5.4 \%$ for non- reactive patients. Therefore the HIV status seemed not to be a factor in developing late toxicities. In the study those who developed grade 2 bladder toxicity $5(2.5 \%)$ were HIV non- reactive, whereas $2(1.5 \%)$ were reactive, grade $3: 11(5.4 \%)$ were HIV non-reactive, whereas $5(3.8 \%)$ were reactive, Grade 4: $12(5.9 \%)$ were non-reactive whereas $7(5.4 \%)$ were reactive. For rectal grade 1: $12(9.2 \%)$ patients were HIV reactive whereas $11(5.4 \%)$ were non-reactive, grade $2: 33(16.3 \%)$ were non-reactive whereas $18(13.8 \%)$ were reactive, for grade 3 : $1(0.5 \%)$ was non-reactive, whereas $2(1.5 \%)$ was reactive, grade 4 all the $2(1.0 \%)$ patients were non-reactive.

It is also important to note that the findings of this study on the relationship of HIV reactive patients and non reactive patients did not take into account the CD4 count of the patients, viral loads and whether patients were on anti-retroviral therapy or not. The aspect that can be considered in other future studies.

\section{Energies and Late Toxicities}

The results of the energies and the late toxicity grades for rectum was conducted. It was noticed that most of the patients who developed the highest grade of toxicity which is grade 4 for both rectum and bladder at $4 \%$ and $10 \%$ respectively were treated with $10 \mathrm{MV}$. This could be attributed to the fact that photon energies in the ranges of $10 \mathrm{MV}$ and above have potentially a higher exit dose which poses a risk of over dosing normal tissue [16].

\section{Chemotherapy and Late Toxicity}

Chemotherapy and late toxicity grade for bladder and rectum show that the majority of patients who developed late toxicities received chemotherapy as compared to those who did not. Significantly for rectal grade $2: 15.8 \%$ had received chemotherapy whereas $13.7 \%$ did not. Similary bladder grade $2: 2.3 \%$ had received whereas $1.4 \%$ did not. This trend was almost the same in the all grades with an exeption of grade 4 were rectal $0.4 \%$ had received and $1.4 \%$ had not, similary grade 4 bladder $5.4 \%$ had received whereas $6.8 \%$ had not. The trend from this study implies that there were more patients who received chemotherapy and developed late toxicities than those that did not. However this findings does not agree with [17] who carried out a systematic review of acute and late toxicity of concomitant chemo-radiation for cervical cancer. Which revealed that late toxicities are not influenced by the presence or absence of chemotherapy because they are dose radiation dependent and manifests with an increase in time after treatment. Furthermore its important to note that unlike in late toxicities, the early acute toxicities of pelvic radiation are increased with the use of chemotherapy [21]. Patients on chemotherapy are also likely to have haematological and nephrotoxicity if cisplatin regimens are used.

\section{Alpha-Beta and Fractionation}

Alpha-Beta and fractionation were constants with alpha- Beta at 3 Gray representing the recommendation by GEC ESTRO which puts a uniform value to show the response of organs at risk [22]. The fractionation of 2 Gray was constant because all the patients received EBRT with the same fractionation and fractionation as an influence on the biological effects of radiation.

\section{ICRU Rectal and Urinary Bladder Doses}

The findings of this study depicted that the mean brachytherapy rectal and urinary bladder doses at ICRU-B and ICRU-R 
reference points for $\mathrm{CDH}$ between 2009-2013 was 59.8\% representing (4.5Gy) and 62\% (4.6 Gy) respectively. These findings are in line with the recommendations by ICRU 38 which states that the maximum dose to the rectum and bladder should be lower than $80 \%$ of the prescribed dose to point $A[4]$.

\section{Rectal and Urinary Bladder Toxicities}

The late toxicities that patients presented with within the period that was understudy were as follows: for rectal the most common were grade 3 at $15.3 \%$ and grade 1 at $6.9 \%$. Whereas for urinary bladder the late toxicities presented were in the following category grade 4 at $6 \%$, grade 3 at $5 \%$ and grade 2 at $2 \%$ respectively. The findings are consistent and within the range with the study done by [14] were it was reported that the chances of developing any late toxicities of any grade in the first five years were at $16.6 \%$ for rectum and $15.7 \%$ for bladder.The number of patients who developed late rectal toxicities were $23.7 \%$ and $(76.3 \%)$ did not. For the bladder only $12.6 \%$ out of 333 developed toxicities. The percentage of patients who did not develop late toxicities are most of the time less than those that develop toxicities as evidenced in the study done by Kim et al. [6] were $57.4 \%$ did not and 42.6 $\%$ developed late toxicities. However the number of patients developing toxicities can even reduce further if conformal image guided $3 \mathrm{D} B \mathrm{~T}$ is used, this is so because the dose reaching the tumour will be maximised and dose reaching the critical organs is minimised [23].

\section{Correlation of Toxicities to Dose}

In the correlation analysis undertaken results did not show a clear relationship between the calculated doses and the manifested toxicity. For example as the (EDQ2) increased from 94.5Gy to $102.8 \mathrm{~Gy}$, the late toxicity grades seeing were from 1-4 for rectum. However as EDQ2 increased beyond $102.8 \mathrm{~Gy}$, there were no toxicities seen, with an exception of 1 case at $120.4 \mathrm{~Gy}$. At high EQD2 it was expected that more rectal toxicities would have been developed, furthermore for the bladder late toxicity grades 2-4 were present at $94.5 \mathrm{~Gy}$ and $102.8 \mathrm{~Gy}$, and beyond EQD2 102.8 Gy there were no late toxicities seen.

The study revealed that there was no correlation between the brachytherapy doses and the late rectal toxicities. For the bladder toxicities the study failed to reject the alternative hypothesis that there was no association between EQD2 and late toxicity grade. This could be attributed to the lack of dose volume relationships employed when using ICRU reference points, plain radiographs are taken and the dose prescribed to point $\mathrm{A}$ [4].

\section{CONCLUSION}

There was no correlation between EQD2 (EBRT + BT) and late rectal toxicities in patients treated with definitive radiotherapy of uterine cervical cancer, and the study also failed to reject the alternative hypothesis that there was association between EDQ2 and late toxicity grade for bladder.

ICRU reference points cannot be reliably be used to demonstrate dose organs relationship and prediction of radiation toxicities to organs at risk. However the point dose methods can still be used in areas where they cannot afford 3D equipment. In 3D the use of dose volume histograms helps in evaluating the doses that critical organs receive and ensure that they are not exceeding the tolerance doses.

The statement is supported by the study findings were the mean brachytherapy and rectal doses were found to be below the maximum dose to the rectum and bladder which should be below $80 \%$ of the prescribed dose to point $\mathrm{A}$. The mean dose for rectum and bladder was $59.8 \%(4.5 \mathrm{~Gy})$ and $62 \%(4.5 \mathrm{~Gy})$ respectively below the ICRU 38 recommendation. Furthermore the prevalence of late toxicities were lower for both rectum and bladder at $23.7 \%$ and $12.6 \%$ respectively.

The study also established that the chances of developing any toxicities of any grade in first 24 months were $15.3 \%$ for rectum and $6 \%$ for urinary bladder. The study further revealed that the number of patients who develop late toxicities are less than those that do not. In this study only $23.7 \%$ and $12.6 \%$ developed rectal and bladder toxicities respectively and the rest did not. The age of the patients was not a factor in developing late toxicities as the prevalence of toxicity were evenly distributed. The study revealed that been HIV positive did not influence the outcome of the toxicities. The study had more patients who were HIV non reactive who developed toxicities as compared to the reactive ones. The study revealed that out of the 79 who developed late rectal toxicity they were 48 non-reactive patients as compared to 31 who were reactive. For bladder also out of the 42 who developed late bladder toxicity 28 patients were non reactive patients as compared to 14 who were reactive.

\section{RECOMMENDATIONS}

- Transitioning of $2 \mathrm{D}$ to $3 \mathrm{D}$ should be encouraged this is due to the fact that $3 \mathrm{D}$ is more superior to $2 \mathrm{D}$ treatment planning and there is dose organ volume relantionship in $3 \mathrm{D}$. Literature also shows that theres is limitation in the use of ICRU dose reporting points. Since dose is prescribed to a point and not to the volume of the tumour.

- There is also need to emphasise to the radiation oncologist and registrars on the need to consistently and accurately document radiation induced toxicities during patients followups as this is one of the indicators that shows patients response to treatment.

- Much work needs to be done on storing information for future use,In the hard copy files vital patients information is lost as loose 
papers are misplaced. An introduction of electronic files for patients can go along way to safeguard the information for longer time.

- There is need for a longitidunal prospective study to be conducted in future that will consistently and accurately record late toxicities on patients followups. This will help in ensuring that the late toxicities are captured at the right time and recorded propely.

- Some of the radiation oncologist and registrars handwriting are difficult to read when the patient's files are hard copies and hand written. So that the next person can be able to use the information for the benefit of managing the patient, education and research that will help in improving treatment delivery. Typed patient information for follow up visits can be considered as an alternative.

\section{REFERENCES}

1. Banerjee, R., \& Kamrava, M. (2014). Brachytherapy in the treatment of cervical cancer: a review. International journal of women's health, 6, 555.

2. Vargo, J. A., \& Beriwal, S. (2014). Image-based brachytherapy for cervical cancer. World journal of clinical oncology, 5(5), 921.

3. Viswanathan, A. N., Beriwal, S., Jennifer, F., Demanes, D. J., Gaffney, D., Hansen, J., ... \& Erickson, B. (2012). American Brachytherapy Society consensus guidelines for locally advanced carcinoma of the cervix. Part II: high-dose-rate brachytherapy. Brachytherapy, 11(1), 47-52.

4. ICRU, (1985). International commission of radiation units and measurement of dose and volume specification for reporting intracavitary therapy in gynaecology. Bethesda Report 38.

5. Viswanathan, A. N., \& Erickson, B. A. (2010). Three-dimensional imaging in gynecologic brachytherapy: a survey of the American Brachytherapy Society. International Journal of Radiation Oncology* Biology* Physics, 76(1), 104-109.

6. Kim, H. J., Kim, S., Ha, S. W., \& Wu, H. G. (2008). Are doses to ICRU reference points valuable for predicting late rectal and bladder morbidity after definitive radiotherapy in uterine cervix cancer?. Tumori Journal, 94(3), 327-332.

7. Chandel, S. S., \& Jain, R. K. (2016). Evaluation of role of radical radiotherapy and chemotherapy in cervical cancer patients-A preliminary report. Clinical Cancer Investigation Journal, 5(2), 126.

8. Khan, F.M \& Gibbons, J.P. (2014). The Physics of Radiation Therapy (5 $5^{\text {th }}$ ed). Lippincott Williams \& Wilkins. Philadelphia

9. Kehwah \& Manish. (2016). High dose rate brachytherapy of carcinoma of the cervix: Applicability of various dosimetry systems and guidelines in the prescription and treatment planning. Austin Journal Oncology \& Cancer. 2(1):1-5.

10. Shadad A.K. (2013). Gastrointestinal radiation injury: Prevention and Treatment. 19 (2), 199-208.

11. Mann, C. J. (2003). Observational research methods. Research design II: cohort, cross sectional, and case-control studies. Emergency medicine journal, 20(1), 54-60.

12. Radiation Therapy Oncology Group. (1999). RTOG/EORTC late radiation morbidity scoring schema. http://www. rtog. org/members/toxicity/late. html.

13. Gattoc, L. (2015).Cervical Cancer/ CancerManagement. Retrieved 18 November, 2016 from www.cancernetwork.com/cancermanagement/cervical.

14. Zobec, Logar, H.B. (2013). Definitive radiotherapy for uterine cervix cancer: long term results for patients treated in the period from 1998 till 2002 at the institute of Oncology Ljubljana. Radiol Oncology, 47(3): 280-288.

15. Edward, C. (2013). Principles and Practice of radiation oncology (6 th ed). Wolters Kluwer/ Lippincott Williams \& Wilkins. Philadelphia.

16. Das, I. J., \& Kase, K. R. (1992). Higher energy: is it necessary, is it worth the cost for radiation oncology?. Medical physics, 19(4), 917-925.

17. Kirwan, J.M. (2003). A systematic review of acute and late toxicity of concomitant chemo-radiation for cervical cancer. Radiotherapy Oncology. 68 (3) 26-217.

18. Huang, E. Y., Sung, C. C., Ko, S. F., Wang, C. J., \& Yang, K. D. (2007). The different volume effects of small-bowel toxicity during pelvic irradiation between gynecologic patients with and without abdominal surgery: a prospective study with computed tomography-based dosimetry. International Journal of Radiation Oncology* Biology* Physics, 69(3), 732-739.

19. Parker, W. I. L. L. I. A. M., \& Patrocinio, H. O. R. A. C. I. O. (2005). Clinical treatment planning in external photon beam radiotherapy. Radiation oncology physics: A handbook for teachers and students. Vienna: IAEA, 219.

20. Mdletshe, S., Munkupa, H., \& Lishimpi, K. (2016). Acute toxicity in cervical cancer HIV-positive vs. HIV-negative patients treated by radical chemo-radiation in Zambia. Southern African Journal of Gynaecological Oncology, 8(2), 37-41.

21. Kavanagh, B. D., Pan, C. C., Dawson, L. A., Das, S. K., Li, X. A., Ten Haken, R. K., \& Miften, M. (2010). Radiation dose-volume effects in the stomach and small bowel. International Journal of Radiation Oncology* Biology Physics, 76(3), S101-S107.

22. De Leeuw, A. A., Van de Kamer, J. B., Moerland, M. A., Philippens, M. E., \& Jürgenliemk-Schulz, I. M. (2011). The effect of alternative biological modelling parameters $(\alpha / \beta$ and half time of repair $T 1 / 2)$ on reported EQD2 values in the treatment of advanced cervical cancer. Radiotherapy and Oncology, 101(2), 337-342.

23. IAEA. (2015). The transition from $2 \mathrm{D}$ brachytherapy to 3D high dose rate brachytherapy. IAEA human reports no 12 . Retrieved 20 $0^{\text {th }}$ May 2016 from pub.iaea.org/MTCD/publications/ PDF/pub 1681 web-80878722.pdf.

24. SEER cancer statistics fact sheets. (2006-2012). Cervical uteri cancer. Retrieved 19 November 2016 from http://seer.cancer.gov/statfacts/html. 